\title{
Suppression of Zeeman Relaxation in Cold Collisions of $\left.\backslash{ }^{\wedge} 2 P \_\{1 / 2\} \backslash\right)$ Atoms
}

\section{Citation}

Tscherbul, T. V., A. A. Buchachenko, Alexander Dalgarno, M.-J. Lu, J. D. Weinstein. 2009. Suppression of Zeeman relaxation in cold collisions of $\backslash\left({ }^{\wedge} 2 \mathrm{P} \_\{1 / 2\} \backslash\right)$ atoms. Physical Review $A$ 80:040701(R).

\section{Published Version}

doi:10.1103/PhysRevA.80.040701

\section{Permanent link}

http://nrs.harvard.edu/urn-3:HUL.InstRepos:10058473

\section{Terms of Use}

This article was downloaded from Harvard University's DASH repository, and is made available under the terms and conditions applicable to Other Posted Material, as set forth at http:// nrs.harvard.edu/urn-3:HUL.InstRepos:dash.current.terms-of-use\#LAA

\section{Share Your Story}

The Harvard community has made this article openly available.

Please share how this access benefits you. Submit a story.

\section{Accessibility}




\title{
Suppression of Zeeman relaxation in cold collisions of ${ }^{2} P_{1 / 2}$ atoms
}

\author{
T. V. Tscherbul, ${ }^{1,2}$ A. A. Buchachenko, ${ }^{3}$ A. Dalgarno,,${ }^{1,2}$ M.-J. Lu, ${ }^{4}$ and J. D. Weinstein ${ }^{4}$ \\ ${ }^{1}$ Harvard-MIT Center for Ultracold Atoms, Cambridge, Massachusetts 02138, USA \\ ${ }^{2}$ Institute for Theoretical Atomic, Molecular, and Optical Physics, Harvard-Smithsonian Center for Astrophysics, Cambridge, \\ Massachusetts 02138, USA \\ ${ }^{3}$ Department of Chemistry, Moscow State University, Moscow 119991, Russia \\ ${ }^{4}$ Department of Physics, University of Nevada, Reno, Nevada 89557, USA
}

(Received 6 July 2009; published 1 October 2009)

\begin{abstract}
We present a combined experimental and theoretical study of angular momentum depolarization in cold collisions of ${ }^{2} \mathrm{P}$ atoms in the presence of an external magnetic field. We show that collision-induced Zeeman relaxation of $\mathrm{Ga}\left({ }^{2} P_{1 / 2}\right)$ and $\operatorname{In}\left({ }^{2} P_{1 / 2}\right)$ atoms in cold ${ }^{4} \mathrm{He}$ gas is dramatically suppressed compared to atoms in ${ }^{2} P_{3 / 2}$ states. Using rigorous quantum-scattering calculations based on $a b$ initio interaction potentials, we demonstrate that Zeeman transitions in collisions of atoms in ${ }^{2} P_{1 / 2}$ electronic states occur via couplings to the ${ }^{2} P_{3 / 2}$ state induced by the anisotropy of the interaction potential. Our results suggest the feasibility of sympathetic cooling and magnetic trapping of ${ }^{2} P_{1 / 2}$-state atoms, such as halogens, thereby opening up exciting areas of research in precision spectroscopy and cold-controlled chemistry.
\end{abstract}

DOI: 10.1103/PhysRevA.80.040701

PACS number(s): 34.50.- s, 32.60.+i, 34.20.Cf

For over a decade, ultracold atomic gases have served as a unique platform for exploring new states of matter [1], implementing scalable algorithms for quantum information processing [2], and simulating quantum condensed-matter systems [3]. Although most of the research focused on the alkali-metal atoms, there has been growing interest in cooling and trapping of the alkali-earth and rare-earth atoms [4], transition metals [5], and nonmetals [6]. Ultracold samples of these elements offer a wealth of research applications, ranging from ultraprecise atomic clocks [4] to studies of dipolar quantum matter [7] to novel ideas for quantum information processing [8]. Whether or not these ideas will be realized in experiments depends on the availability of efficient methods for cooling and trapping of non- $S$-state atoms.

The experimental technique of buffer gas cooling relies on elastic collisions with cryogenic He gas to cool paramagnetic atoms to temperatures below $1 \mathrm{~K}$ and load them in a permanent magnetic trap $[5,6]$. The atoms are confined in low-field-seeking Zeeman states, which are intrinsically unstable and may undergo Zeeman relaxation in collisions with background $\mathrm{He}$ atoms, causing trap loss. The lifetime of trapped atoms is determined by the ratio $\gamma$ of the cross sections for elastic and inelastic collisions. The ratio must be large enough $\left(\gamma>10^{4}\right)$ to allow for efficient cryogenic cooling and trap loading. The detrimental inelastic collisions occur due to couplings between different Zeeman levels induced by the anisotropy of the interaction potential between the atoms [9-11]. Previous experimental and theoretical work has shown that anisotropic interactions in collisions of $P$-state atoms, such as $\mathrm{Ca}\left({ }^{3} P_{2}\right)$ or $\mathrm{Yb}\left({ }^{3} P_{2}\right)$, are strong $(\gamma \sim 1)$, leading to the belief that sympathetic cooling of non- $S$-state atoms in a magnetic trap would not be successful $[10,12]$.

Here, we present a joint experimental and theoretical study of low-temperature collisions of $\mathrm{Ga}$ and In atoms in a magnetic field. We find that inelastic transitions in collisions of ${ }^{2} P_{1 / 2}$ atoms with ${ }^{4} \mathrm{He}$ are dramatically suppressed. Using rigorous quantum calculations based on $a b$ initio interaction potentials, we show that the suppression is common to all ${ }^{2} P_{1 / 2}$ atoms. Our results suggest the possibility for sympathetic cooling and magnetic trapping of ${ }^{2} P_{1 / 2}$ atoms, with potential applications in precision spectroscopy, quantum optics, and cold-controlled chemistry [13].

Our experimental apparatus is similar to that described in Ref. [14]. Ga(In) atoms are produced by laser ablation of $\mathrm{Ga}-\mathrm{Cu}$ alloy (In metal) targets, and subsequently cooled to a translational temperature of $5 \mathrm{~K}$ with a cryogenic ${ }^{4} \mathrm{He}$ buffer gas. Laser absorption spectroscopy on the $\left[n s^{2} n p\right]^{2} P_{1 / 2}^{\circ} \rightarrow\left[n s^{2}(n+1) s\right]^{2} S_{1 / 2}$ transitions at 410 and 403 $\mathrm{nm}$ is used to state-selectively monitor the ground finestructure-state populations of In and Ga, respectively [15]. A typical spectrum of atomic Ga is shown in Fig. 1(a). The low translational temperature allows us to spectrally resolve the isotopes and hyperfine states. By measuring the diffusion lifetime as a function of helium density, we determine the thermally averaged diffusion cross section of $\mathrm{Ga}$ to be $(7.5 \pm 2.0) \times 10^{-15} \mathrm{~cm}^{2}$ and of In $(9.1 \pm 2.5) \times 10^{-15} \mathrm{~cm}^{2}$.

To measure inelastic collisions, the internal-state distribution of the atoms is perturbed by optical pumping on the same transitions as used for absorption spectroscopy [16]. By monitoring the return of the atomic population to equilibrium, we determine the rates for $F$-changing and $J$-changing transitions in ${ }^{69} \mathrm{Ga}-{ }^{4} \mathrm{He}$ collisions, where $J$ is the total angular momentum $F$ of the atom exclusive of nuclear spin $I$ $\left(I_{\mathrm{Ga}}=3 / 2\right.$ and $\left.I_{\mathrm{In}}=9 / 2\right)$, and we obtain upper bounds to the relaxation rates for ${ }^{115} \mathrm{In}-{ }^{4} \mathrm{He}$ collisions.

Figure 1(b) shows the time dependence of the optical density for ${ }^{69} \mathrm{Ga}$ atoms in the $J=1 / 2, F=2$ hyperfine state. Prior to the pump pulse, there is no measurable polarization in the atoms. After pumping a large polarization is induced, as indicated by the relative absorption of $\sigma^{+}$and $\sigma^{-}$light. This polarization decays over time. By fitting the difference in the optical densities to the functional form $e^{-t / \tau}$ and repeating this measurement over a range of helium densities $n$, we determine the effective $m$-changing rate coefficient from $n k_{m}=1 / \tau$. The dependence of $\tau$ on the helium density indi- 
(a)
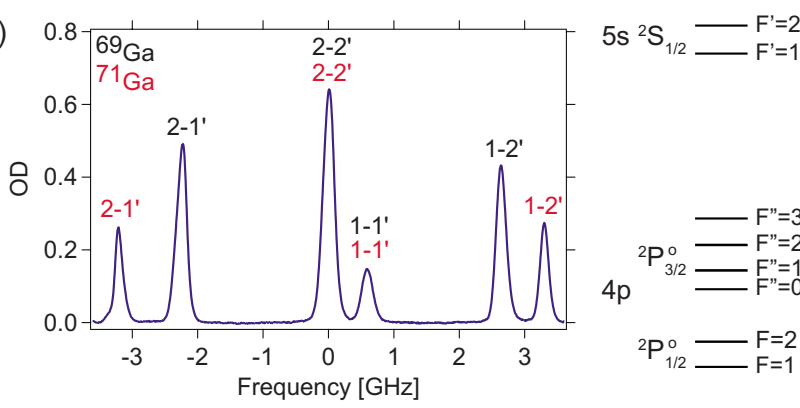

(b)

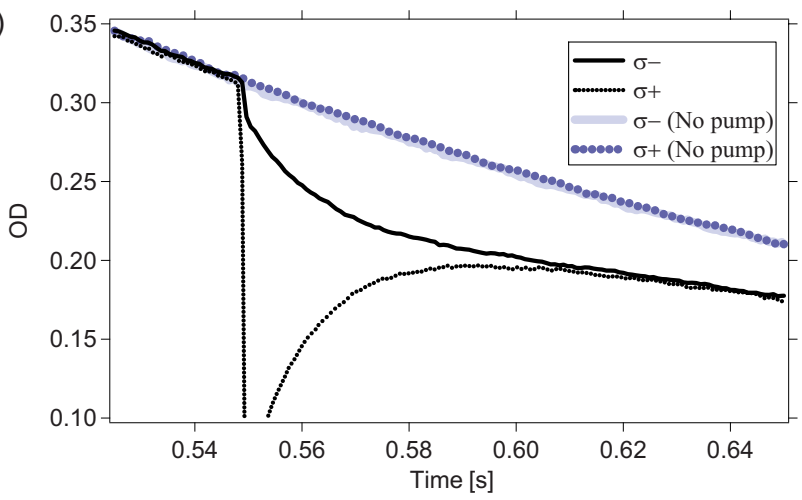

FIG. 1. (Color online) (a) Absorption spectrum of ${ }^{69} \mathrm{Ga}\left({ }^{2} P_{1 / 2}^{\circ}\right)$. The peaks are labeled according to their isotope and hyperfine transitions. (b) Optical density of ${ }^{69} \mathrm{Ga}$ as observed by $\sigma^{+}$and $\sigma^{-}$probe beams on the $F=2 \rightarrow 1$ transition at a helium density of $6.4 \times 10^{16} \mathrm{~cm}^{-3}$ and a bias field of $3 \mathrm{G}$. The ablation laser fires at $t=0$ and a strong $\sigma^{+}$pump beam is turned on for $1 \mathrm{~ms}$ at $t=0.55 \mathrm{~s}$. Also shown is the optical density in the absence of optical pumping; its level is renormalized to compensate for shot-toshot inconsistencies in ablation yield.

cated the presence of additional relaxation mechanisms, and so we could only place upper limits on the $m$-changing rates listed in Table I.

After optical pumping, as shown in Fig. 1(b), the overall population in the pumped hyperfine state is reduced. This population returns to equilibrium on a time scale longer than is shown in Fig. 1. By fitting the return to equilibrium to an exponential function, we find time constants $T_{F}$ and $T_{J}$ for $F$-changing and $J$-changing collisions. We can distinguish

TABLE I. Experimental and theoretical rate constants for Zeeman relaxation of ${ }^{69} \mathrm{Ga}$ and ${ }^{115} \mathrm{In}$ atoms in the $|J=1 / 2, F=J+I, m=F\rangle$ hyperfine states by collisions with ${ }^{4} \mathrm{He}$ at $T=5 \mathrm{~K}$ and $B=3 \mathrm{G}$ (in units of $10^{-17} \mathrm{~cm}^{3} / \mathrm{s}$ ). Also shown are the ratios of the rate constants for diffusion and inelastic relaxation $\gamma$. The values in parentheses are calculated with the interaction anisotropy multiplied by 1.2 .

\begin{tabular}{|c|c|c|c|c|}
\hline \multirow{2}{*}{$\begin{array}{l}\text { Atom } \\
\text { Rate constant }\end{array}$} & \multicolumn{2}{|c|}{$\mathrm{Ga}$} & \multicolumn{2}{|c|}{ In } \\
\hline & Exp. & Theory & Exp. & Theory \\
\hline$k_{m}$ & $<300$ & $0.8(2.3)$ & $<50$ & 3.8 \\
\hline$k_{F}$ & $5.3 \pm 1.3$ & $2.3(6.6)$ & $<2.3 \pm 1.4$ & 0.1 \\
\hline$k_{J}$ & $1.0 \pm 0.3$ & 0.03 & $<8$ & 0.0004 \\
\hline$\gamma$ & $>4.2 \times 10^{4}$ & $\left(2.6 \times 10^{6}\right)$ & $>3.0 \times 10^{5}$ & $6.7 \times 10^{6}$ \\
\hline
\end{tabular}

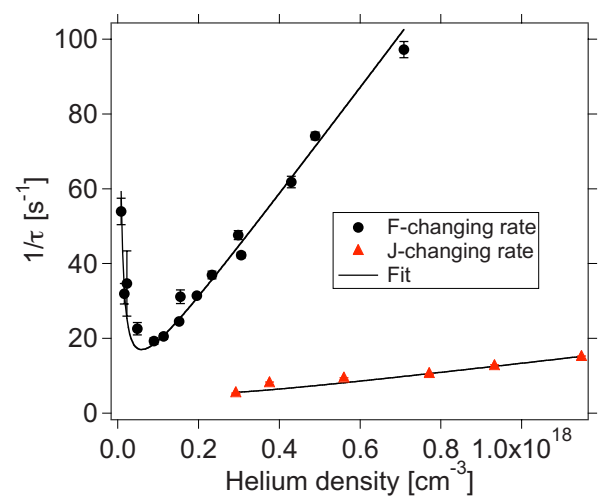

FIG. 2. (Color online) Measured ${ }^{69} \mathrm{Ga} F$ - and $J$-relaxation rates $1 / \tau$ for different ${ }^{4} \mathrm{He}$ densities.

the two processes by monitoring the population of both hyperfine states in the $J=1 / 2$ manifold. To determine the rate coefficients $k$, we measure these rates over a range of $n$, as shown in Fig. 2, and fit our data to the form $1 / \tau=k n+C / n$, where the term $C / n$ accounts for the diffusion of atoms in and out of the probe beam.

In order to interpret the experimental observations, we extended the quantum-scattering formalism of Ref. [11] to describe collisions of ${ }^{2} P$ atoms with nonzero nuclear spins. The Hamiltonian of the $M\left({ }^{2} P\right)$-He collision complex may be written in atomic units as [11]

$\hat{H}=-\frac{1}{2 \mu R} \frac{\partial^{2}}{\partial R^{2}} R+\frac{\hat{\ell}^{2}}{2 \mu R^{2}}+\hat{H}_{M}+\hat{V}(\boldsymbol{R}, \boldsymbol{r})+\left[A(R)-A_{\mathrm{SO}}\right] \hat{L} \cdot \hat{S}$,

where $\mu$ is the reduced mass of the complex, $\hat{\ell}$ is the orbital angular momentum for the collision, $A(R)$ is the spin-orbit (SO) constant of the $M$-He complex, and $\hat{V}(\boldsymbol{R}, \boldsymbol{r})$ is the atomatom interaction potential as a function of interatomic separation $(\boldsymbol{R})$ and electronic coordinates $(\boldsymbol{r})$. The Hamiltonian of the isolated atom $M$ in a magnetic field is

$$
\begin{aligned}
\hat{H}_{M}= & A_{\mathrm{SO}} \hat{L} \cdot \hat{S}+a_{J} \hat{I} \cdot \hat{J}+2 b_{J} \hat{I} \cdot \hat{J}(2 \hat{I} \cdot \hat{J}+1)+\mu_{0} B\left(\hat{L}_{z}+2 \hat{S}_{z}\right) \\
& -B \frac{\mu_{I}}{I} \hat{I}_{z}
\end{aligned}
$$

where $\hat{L}$ is the electronic orbital angular momentum and $\hat{S}$ is the electron spin. The SO, Fermi hyperfine, and nuclear electric quadrupole interactions are parametrized by the constants $A_{\mathrm{SO}}=2 \Delta / 3, a_{J}$, and $b_{J}[17-19]$, where $\Delta$ is the atomic SO splitting (see Table II). The interaction of the atom with a magnetic field of strength $B$ is given by $\mu_{0} B\left(\hat{L}_{z}+2 \hat{S}_{z}\right)-B \frac{\mu_{I}}{I} \hat{I}_{z}$, where $\mu_{0}$ is the Bohr magneton and $\mu_{I}$ is the nuclear magnetic moment. We expand the wave function of the collision complex in the uncoupled basis $\left|J m_{J}\right\rangle\left|m_{I}\right\rangle\left|\ell m_{\ell}\right\rangle$, where $m_{J}, m_{I}$, and $m_{\ell}$ are the projections of $\hat{J}, \hat{I}$, and $\hat{\ell}$ on the magnetic field axis. Inserting the expansion into Eq. (1) leads to a system of close-coupled differential equations, which we solve to obtain the probabilities for collision-induced Zeeman transitions $|J F m\rangle \rightarrow\left|J^{\prime} F^{\prime} m^{\prime}\right\rangle$, 
TABLE II. The calculated ratios of the rate constants for elastic scattering and Zeeman relaxation in collisions of $\mathrm{Al}, \mathrm{Ga}$, In, and halogen atoms with ${ }^{3} \mathrm{He}$ at $T=0.5 \mathrm{~K}$ and $B=0.5 \mathrm{~T}$.

\begin{tabular}{lrcc}
\hline \hline & \multicolumn{1}{c}{$\Delta$} & & \\
Atom & $\left(\mathrm{cm}^{-1}\right)$ & $\gamma(J=1 / 2)$ & $\gamma(J=3 / 2)$ \\
\hline $\mathrm{F}$ & 404.14 & $1.9 \times 10^{2}$ & 4.7 \\
$\mathrm{Cl}$ & 882.35 & $5.0 \times 10^{4}$ & 2.7 \\
$\mathrm{Br}$ & 3685.24 & $1.3 \times 10^{8}$ & 3.0 \\
$\mathrm{I}$ & 7603.15 & $1.6 \times 10^{9}$ & 3.0 \\
$\mathrm{Al}$ & 112.06 & $7.0 \times 10^{3}$ & 2.4 \\
$\mathrm{Ga}$ & 826.19 & $4.1 \times 10^{6}$ & 33.0 \\
$\mathrm{In}$ & 2212.60 & $4.6 \times 10^{7}$ & 15.1 \\
\hline \hline
\end{tabular}

where $m=m_{J}+m_{I}$. We identify three main contributions to the total Zeeman relaxation rate arising from the $m$-changing $(\Delta J=\Delta F=0), F$-changing $(\Delta J=0)$, and $J$-changing $(\Delta J \neq 0)$ transitions. The matrix elements of the interaction potential in Eq. (1) are [11]

$$
\begin{aligned}
& \left\langle J m_{J}\left|\left\langle\operatorname{Im}_{I}\left|\left\langle\ell m_{\ell}|\hat{V}(\boldsymbol{R}, \boldsymbol{r})| J^{\prime} m_{J}^{\prime}\right\rangle\right| \operatorname{Im}_{I}^{\prime}\right\rangle\right| \ell^{\prime} m_{\ell}^{\prime}\right\rangle \\
& =\delta_{m_{I} m_{I}^{\prime}}(-)^{S+J+J^{\prime}-m_{J}-m_{\ell}^{\prime}}\left[(2 L+1)(2 L+1)(2 J+1)\left(2 J^{\prime}+1\right)\right. \\
& \left.\times(2 \ell+1)\left(2 \ell^{\prime}+1\right)\right]^{1 / 2} \sum_{\lambda=0,2} V_{\lambda}(R)\left\{\begin{array}{ccc}
L & J & S \\
J^{\prime} & L & \lambda
\end{array}\right\} \\
& \times\left(\begin{array}{ccc}
J & \lambda & J^{\prime} \\
-m_{J} & m_{J}-m_{J}^{\prime} & m_{J}^{\prime}
\end{array}\right)\left(\begin{array}{ccc}
\ell & \lambda & \ell^{\prime} \\
-m_{\ell} & m_{\ell}-m_{\ell}^{\prime} & m_{\ell}^{\prime}
\end{array}\right) \\
& \times\left(\begin{array}{ccc}
L & \lambda & L \\
0 & 0 & 0
\end{array}\right)\left(\begin{array}{lll}
\ell & \lambda & \ell^{\prime} \\
0 & 0 & 0
\end{array}\right),
\end{aligned}
$$

where the symbols in parentheses and figure brackets are $3-j$ and $6-j$ symbols, and $V_{\lambda}$ 's are the isotropic $\left(V_{0}\right)$ and anisotropic $\left(V_{2}\right)$ parts of the interaction potential of the $M\left({ }^{2} P\right)$-He complex [11].

To evaluate the interaction potentials, we used the coupled cluster method with single, double, and noniterative triple excitations as implemented in the MOLPRO suite of programs [20]. For Ga and In, we employed relativistic effective core pseudopotentials [21] in combination with extended correlation-consistent aug-cc-pV5Z (AV5Z) basis sets [22], augmented with diffuse functions [spdfgh]. For He, we used a doubly augmented AV5Z basis of similar quality [23]. An additional set of basis functions [ $3 s 3 p 2 d 2 f 1 g]$ was placed at the midpoint of the $M-\mathrm{He}$ bond [24], and the full counterpoise correction procedure was applied to eliminate the basis set superposition error. The SO constants $A(R)$ were evaluated using the multireference configuration-interaction method.

The cross sections for collision-induced Zeeman relaxation of $\mathrm{Ga}$ and In atoms in fully spin-polarized states $|J, F=J+I, m=F\rangle$ are plotted in Fig. 3 as functions of collision energy. The inelastic relaxation of atoms in ${ }^{2} P_{3 / 2}$ states proceeds almost entirely via $J$-conserving transitions, which occur at a large rate comparable to that for elastic energy transfer [10]. In contrast, Zeeman transitions in collisions of

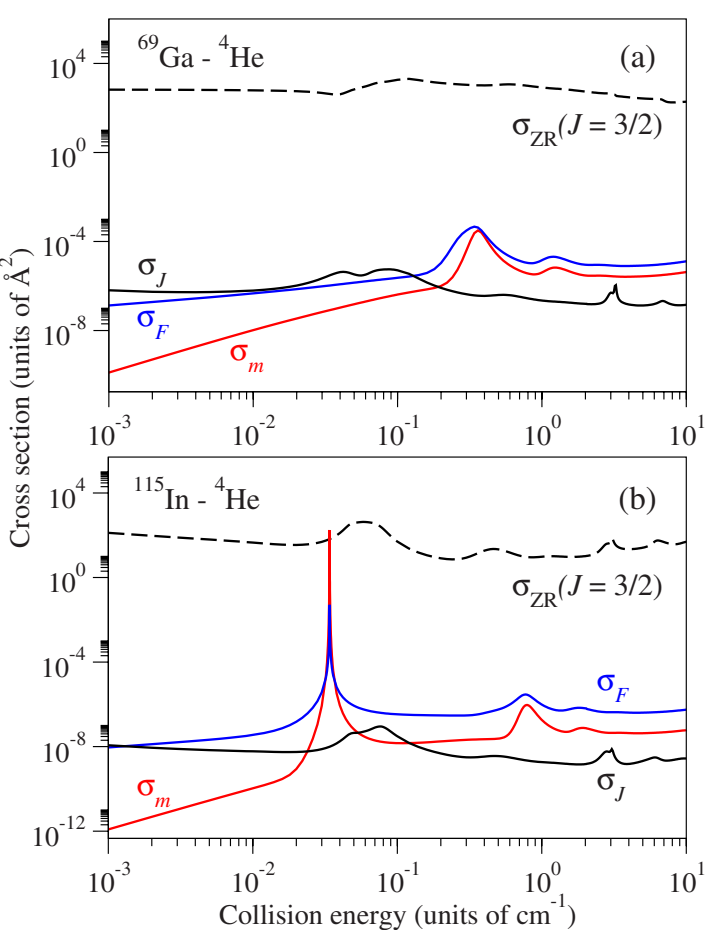

FIG. 3. (Color online) The cross sections for $m$-changing $\left(\sigma_{m}\right)$ and $F$-changing $\left(\sigma_{F}\right)$ transitions in $M\left({ }^{2} P_{1 / 2}\right)-{ }^{4} \mathrm{He}$ collisions with (a) $M={ }^{69} \mathrm{Ga}$ and (b) ${ }^{115}$ In calculated as functions of collision energy at a magnetic field of $3 \mathrm{G}$. Also shown are the cross sections for collision-induced Zeeman relaxation of ${ }^{2} P_{3 / 2}$ atoms defined as $\sigma_{\mathrm{ZR}}=\sigma_{m}+\sigma_{F}+\sigma_{J}$, where $\sigma_{J}$ is the cross section for the transition $J=3 / 2 \rightarrow J=1 / 2$.

${ }^{2} P_{1 / 2}$-state $\mathrm{Ga}$ and $\mathrm{In}$ atoms are strongly suppressed over a wide range of collision energies. Table I presents the calculated and measured rate constants for Zeeman relaxation of $\mathrm{Ga}$ and In atoms in a buffer gas of ${ }^{4} \mathrm{He}$ at $T=5 \mathrm{~K}$. Although the calculated $m$-changing rates are consistent with the measured upper bounds for both $\mathrm{Ga}-{ }^{4} \mathrm{He}$ and $\mathrm{In}-{ }^{4} \mathrm{He}$, the theoretical rates for $F$-changing and $J$-changing transitions for $\mathrm{Ga}^{4} \mathrm{He}$ are too small, which indicates that our ab initio calculations may underestimate the $\mathrm{Ga}-\mathrm{He}$ interaction anisotropy. Table I shows that the increase in the interaction anisotropy leads to quantitative agreement of both $F$-changing and $J$-changing rates with experimental measurements. The calculated elastic-to-inelastic ratios $\gamma$ are large and consistent with the measured lower bounds. Thus, both experiment and theory suggest that cryogenic cooling and magnetic trapping of $\mathrm{Ga}$ and In atoms at buffer gas densities $n \sim 10^{15} \mathrm{~cm}^{-3}$ would be efficient.

An analysis of Eq. (3) shows that Zeeman transitions in collisions of ${ }^{2} P$ atoms occur due to couplings between different $m_{J}$ levels induced by the anisotropic part of the interaction potential [11]. Because the first $3-j$ symbol in Eq. (3) vanishes for $J=J^{\prime}=1 / 2$, different Zeeman states within the $J=1 / 2$ manifold are not coupled. In contrast, the levels within the $J=3 / 2$ manifold and those in different $J$ manifolds are directly coupled by the anisotropy of the interaction potential. As a result, Zeeman relaxation in collisions of ${ }^{2} P_{1 / 2}$ atoms occurs indirectly via couplings to the ${ }^{2} P_{3 / 2}$-excited state. As shown in Fig. 3, this process slows down dramati- 
cally with an increase in the energy separation between the ${ }^{2} P_{1 / 2}$ and ${ }^{2} P_{3 / 2}$ terms (test calculations for $\mathrm{Ga}-{ }^{4} \mathrm{He}$ show that $\left.k_{m} \sim A_{\mathrm{SO}}^{-4.5}\right)$. For atoms colliding in ${ }^{2} P_{3 / 2}$ states, direct couplings of different $m_{J}$ levels lead to large relaxation rates, which are insensitive to the SO splitting. This result may be qualitatively explained as follows. The electron-density distribution of atoms in ${ }^{2} P_{1 / 2}$ electronic states is spherically symmetric and that of ${ }^{2} P_{3 / 2}$ atoms is not. The interaction with the He atom couples the two fine-structure states and distorts the spherical symmetry of the ${ }^{2} P_{1 / 2}$ state, leading to Zeeman relaxation. This is different for atoms in $J>1 / 2$ states, which undergo Zeeman relaxation through direct couplings within a single fine-structure state.

To determine whether the observed suppression of inelastic collisions occurs for other ${ }^{2} P_{1 / 2}$ atoms, we extended our scattering calculations to include the halogen atoms in both the ground $(J=3 / 2)$ and metastable $(J=1 / 2)$ electronic states using the $a b$ initio interaction potentials from Ref. [25]. Table II shows that elastic-to-inelastic ratios for collisions of spin-polarized $\mathrm{Al}, \mathrm{Ga}$, In, and metastable halogen atoms with ${ }^{3} \mathrm{He}$ are large even in the presence of a strong magnetic field.

In summary, we have presented evidence for low Zeeman relaxation rates in cold collisions of $\mathrm{Ga}\left({ }^{2} P_{1 / 2}\right)$ and $\operatorname{In}\left({ }^{2} P_{1 / 2}\right)$ atoms with both isotopes of $\mathrm{He}$ in a magnetic field. We hope that our results will stimulate research in heretofore unex- plored areas of atomic, molecular, and chemical physics. In particular, Eq. (3) shows that inelastic collisions of ${ }^{2} P_{1 / 2}$ atoms with spin-polarized $S$-state atoms may be similarly suppressed if the atom-atom interaction anisotropy is weak compared to the SO splitting. This suggests that sympathetic cooling of ${ }^{2} P_{1 / 2}$ atoms with alkali-metal atoms in a magnetic trap may be within reach. Cooling and trapping of heavy ${ }^{2} P_{1 / 2}$ atoms (such as $\mathrm{Tl}$ ) may enhance the sensitivity of spectroscopic experiments to measure the electric dipole moment of the electron [26]. An experimental study of cold chemical reactions involving halogen atoms would greatly increase the scope of ultracold chemistry [13] and possibly lead to the realization of the external field control of chemical reactivity at low temperatures.

We acknowledge stimulating discussions with John Doyle and Roman Krems. This work was supported by the Nevada NASA EPSCoR program in Astrophysics, joint Russian Basic Research Fund-Consortium E.I.N.S.T.E.I.N project, and the Chemical Science, Geoscience, and Bioscience Division of the Office of Basic Energy Science, Office of Science, U.S. Department of Energy and NSF grants to the HarvardMIT CUA and ITAMP at Harvard University, and Smithsonian Astrophysical Observatory.
[1] K. Southwell, Nature (London) 416, 205 (2002).

[2] I. Bloch, Nature (London) 453, 1016 (2008).

[3] S. Trotzky et al., Science 319, 295 (2008).

[4] M. M. Boyd et al., Science 314, 1430 (2006).

[5] C. I. Hancox et al., Nature (London) 431, 281 (2004); C. I. Hancox, S. C. Doret, M. T. Hummon, R. V. Krems, and J. M. Doyle, Phys. Rev. Lett. 94, 013201 (2005); N. Brahms et al., ibid. 101, 103002 (2008); A. Hatakeyama et al., ibid. 84, 1407 (2000).

[6] M. T. Hummon et al., Phys. Rev. A 78, 050702(R) (2008).

[7] T. Lahaye et al., Nature (London) 448, 672 (2007).

[8] A. V. Gorshkov et al., Phys. Rev. Lett. 102, 110503 (2009).

[9] R. V. Krems, G. C. Groenenboom, and A. Dalgarno, J. Phys. Chem. A 108, 8941 (2004)

[10] D. Hansen and A. Hemmerich, Phys. Rev. Lett. 96, 073003 (2006); A. Yamaguchi, S. Uetake, D. Hashimoto, J. M. Doyle, and Y. Takahashi, ibid. 101, 233002 (2008).

[11] R. V. Krems and A. Dalgarno, Phys. Rev. A 68, 013406 (2003).

[12] With the exception of atoms whose $d$ - and $f$-electron shells are submerged under the outer filled $s$ shell [5].

[13] R. V. Krems, Phys. Chem. Chem. Phys. 10, 4079 (2008).

[14] M.-J. Lu, K. S. Hardman, J. D. Weinstein, and B. Zygelman, Phys. Rev. A 77, 060701(R) (2008).
[15] O. M. Marago, B. Faxio, P. G. Gucciardi, and E. Arimondo, Appl. Phys. B: Lasers Opt. 77, 809 (2003); H. Leinen et al., Appl. Phys. B 70, 567 (2000).

[16] A. Hatakeyama, K. Enomoto, and T. Yabuzaki, Phys. Scr. T 110, 294 (2004).

[17] The hyperfine constants of $\mathrm{Ga}\left(a_{1 / 2}=1338.99\right.$ and $a_{3 / 2}$ $=190.79 \mathrm{MHz})$ and In $\left(a_{1 / 2}=2283.2, a_{3 / 2}=242.2\right.$, and $b_{3 / 2}$ $=1.56 \mathrm{MHz})$ are borrowed from Refs. $[18,19]$.

[18] S. J. Rosenthal, Phys. Rev. A 21, 248 (1980).

[19] A. K. Mann and P. Kusch, Phys. Rev. 77, 427 (1950).

[20] H.-J. Werner et al., MOLPRO, version 2006.3 (a package of ab initio programs) (Cardiff, UK, 2006).

[21] B. Metz, H. Stoll, and M. Dolg, J. Chem. Phys. 113, 2563 (2000).

[22] K. A. Peterson, J. Chem. Phys. 119, 11099 (2003).

[23] D. E. Woon and T. H. Dunning, Jr., J. Chem. Phys. 100, 2975 (1994).

[24] S. M. Cybulski and R. R. Toczyłowski, J. Chem. Phys. 111, 10520 (1999).

[25] H. Partridge, J. R. Stallcop, and E. Levin, J. Chem. Phys. 115, 6471 (2001).

[26] B. C. Regan, E. D. Commins, C. J. Schmidt, and D. DeMille, Phys. Rev. Lett. 88, 071805 (2002). 